\title{
CHILDHOOD LEUKAEMIA: A RELATIONSHIP BETWEEN INTRACELLULAR 6-MERCAPTOPURINE METABOLITES AND NEUTROPENIA
}

\author{
L. LENNARD, C.A. REES ${ }^{1}$, J.S. LILLEYMAN \& J.L. MADDOCKS \\ University Department of Therapeutics, Royal Hallamshire Hospital, Sheffield and ${ }^{1}$ Department of Haematology, \\ The Children's Hospital, Sheffield
}

1 6-Mercaptopurine is extensively used in the treatment of childhood lymphoblastic leukaemia to prolong the duration of remission achieved with other drugs. The response to remission maintenance therapy varies widely.

2 We investigated the relationship between red blood cell 6-thioguanine nucleotide, a metabolite of 6-mercaptopurine, and myelosuppression in 22 children with acute lymphoblastic leukaemia in remission. The peripheral neutrophil count was used as an index of myelosuppression.

3 6-Mercaptopurine dose was related to 6-thioguanine nucleotide concentration $(r=0.4 ; P<0.001 ; n$ $=90 ; \mathrm{y}=18.51+0.36 \mathrm{x})$. Large individual variations around the regression line are observed.

4 Neither 6-mercaptopurine dose nor 6-thioguanine nucleotide concentration was related to the neutrophil count at the time of sampling (day 0 ) or 7 days later.

5 Both 6-mercaptopurine dose and 6-thioguanine nucleotide concentration correlated with the neutrophil count at day 14 ( $r=-0.33 ; P<0.01 ; n=90$ and $r=-0.3 ; P<0.01 ; n=90$ respectively). This delay is compatible with a cytotoxic action on bone marrow stem cells.

6 Excluding children with other, uncontrolled, potentially myelosuppressive influences the correlation between 6-thioguanine nucleotide concentration and neutropenia improved $(r=-0.6 ; P<0.001 ; n=$ 37). A significant degree of neutropenia was observed by day 14 if the 6-thioguanine nucleotide concentration (day 0 ) was $>210 \mathrm{pmol} / 8 \times 10^{8} \mathrm{RBCs}$.

7 The assay of 6-thioguanine nucleotide may highlight those individuals with pharmacokinetic resistance. Two children on continuous high dose 6-mercaptopurine, had low red blood cell 6-thioguanine nucleotide concentrations and neutropenia was not observed.

Keywords 6-mercaptopurine childhood leukaemia neutropenia

\section{Introduction}

The purine analogue 6-mercaptopurine (6-MP) interferes with cell replication and has antileukaemic and immunosuppressive effects. Although the precise mode of action is not fully understood cytotoxicity is related to the incorporation of 6-MP derived 6-thioguanine (6-TG) nucleotides into DNA (Tidd \& Paterson, 1974). 6-MP is extensively used in children with lymphoblastic leukaemia to prolong the duration of remission achieved with other drugs, but the response to maintenance therapy varies widely. For many children the clinical prospects are inexplicably poor, over half will relapse and die. Interindividual differences in pharmacokinetics may explain some of these unpredictable responses.

Combination chemotherapy is titrated against its effect on the white blood cell count. In a pilot study
(Herber et al., 1982), we observed an inverse correlation between red blood cell (RBC) 6-TG nucleotide at sampling (day 0) and the peripheral neutrophil count $(P<0.01) 14$ days later. This delay in the appearance of the neutropenic effects of 6-MP is compatible with a cytotoxic action on the granulopoietic stem cells of the bone marrow.

The aim of this study was to confirm whether RBC 6-TG nucleotide could be used to predict 6-MP cytotoxicity in a larger group of children with acute lymphoblastic leukaemia in remission. We also examined the rate of 6-TG nucleotide accumulation within the RBC when starting 6-MP remission maintenance therapy.

Part of this work was presented to the British Pharmacological Society at the London Meeting, 1983 (Lennard et al., 1983b). 


\section{Methods}

The patients studied were on the Medical Research Council (MRC) therapeutic trial UKALL VIII, the time on remission maintenance therapy varied from 4 weeks to 2 years. Blood samples $(0.5 \mathrm{ml})$ were taken from 22 unselected children on consecutive clinic visits (90 samples in total). There were 11 boys and 11 girls aged between 2 and 16 years. In addition four children had serial studies from the start of 6-MP therapy. Samples were taken at each clinic visit until steady state 6-TG nucleotide was observed or dose adjustment occurred. Blood samples were taken just before a dose of 6-MP and, when possible, at the time of venepuncture for vincristine therapy. Otherwise full parental consent was obtained.

Remission maintenance therapy consisted of daily 6-MP at a starting dose of $75 \mathrm{mg} / \mathrm{m}^{2}$. In all cases 6-MP doses were adjusted to $75 \%, 50 \%$ or $0 \%$ of the schedule dose on a sliding scale in the face of neutropenia or thrombocytopenia at the time of prescription. Concurrently all patients received a single oral dose of methotrexate $\left(20 \mathrm{mg} / \mathrm{m}^{2}\right)$ weekly, subject to similar dose adjustment, and pulses of prednisone or prednisolone $\left(40 \mathrm{mg} / \mathrm{m}^{2}\right)$ for 5 days every fourth week coupled with a single injection of vincristine (1.5 $\left.\mathrm{mg} / \mathrm{m}^{2}\right)$. The steroid and vincristine dose was constant throughout remission.

RBC 6-TG nucleotide was assayed by the method of Lennard \& Maddocks (1983a). The absolute neutrophil count (ANC) was used as an index of myelosuppression. The ANC was recorded at the time of sampling (day 0), after 7 days and 14 days later. Neutropenia was defined as a reduction in the ANC below the lower normal limit of $1.5 \times 10^{9} \mathrm{I}^{-1}$. Statistical analysis was by Pearson's product-moment correlation coefficient and, for the difference between means, Student's $t$-test. The Fisher-z transformation was used to calculate the $95 \%$ confidence limits for the correlation coefficient $r$. The regression coefficients $a$ and $b$ are expressed \pm s.e. mean. Arithmetical means are expressed as mean \pm s.d.

\section{Results}

\section{Dose and metabolite}

The 6-MP dose ranged from 0 to $83 \mathrm{mg} / \mathrm{m}^{2}$ (mean = $48 \pm 28 \mathrm{mg}$ ). The concentration of RBC 6-TG nucleotide ranged from 0 to 802 pmol per $8 \times 10^{8} \mathrm{RBCs}$ (mean $=210 \pm 149 \mathrm{pmol})$. 6-MP dose related to the RBC 6-TG nucleotide concentration at sampling (day $0)(r=0.4 ; P<0.001 ; n=90)$ (Figure 1$)$, as did the total 6-MP dose for the previous month $(r=0.48 ; P<$ $0.001 ; n=90$ ) (Figure 2).

\section{Myelosuppression}

Neither 6-MP dose nor RBC 6-TG nucleotide concentration correlated with the ANC at day 0 or day 7 . The 6-MP dose (day 0) correlated with the ANC at day $14(r=-0.33 ; P<0.01 ; n=90 ; \mathrm{y}=2.35$ $( \pm 0.29)-0.016( \pm 0.0054) x)$. The $95 \%$ confidence

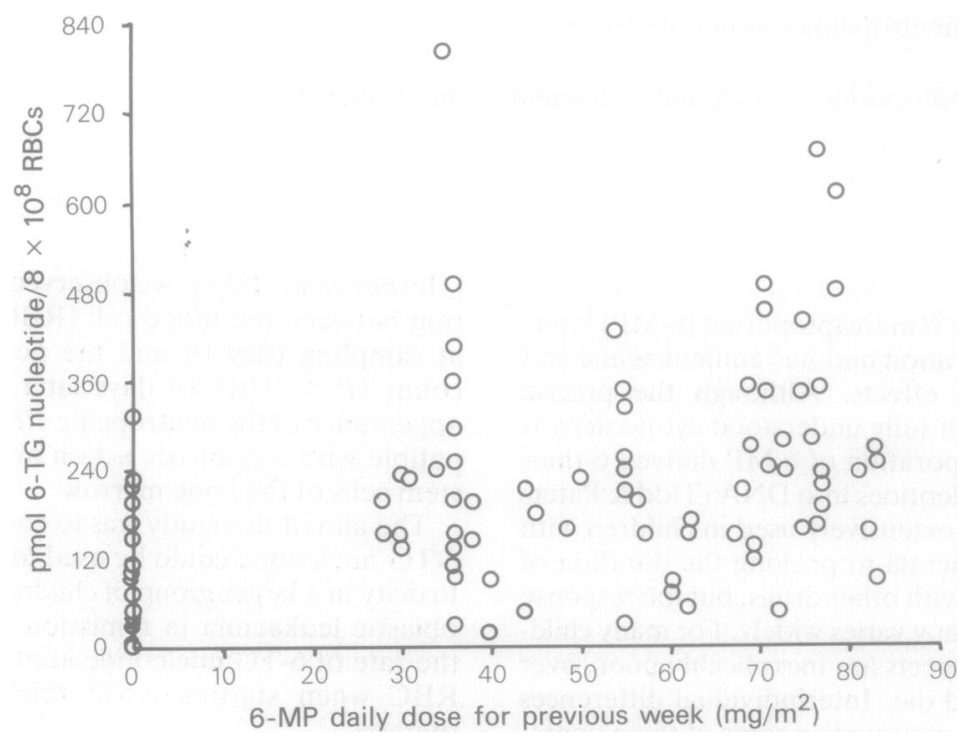

Figure 1 The relationship between 6-mercaptopurine (6-MP) daily dose and 6-thioguanine (6-TG) nucleotide concentration at day $0(r=0.4 ; P<0.001 ; n=90 ; y=18.51( \pm 4.61)+0.36( \pm 0.088) x)$. The $95 \%$ confidence limits for the regression coefficient $b$ are 0.344 to 0.376 . 


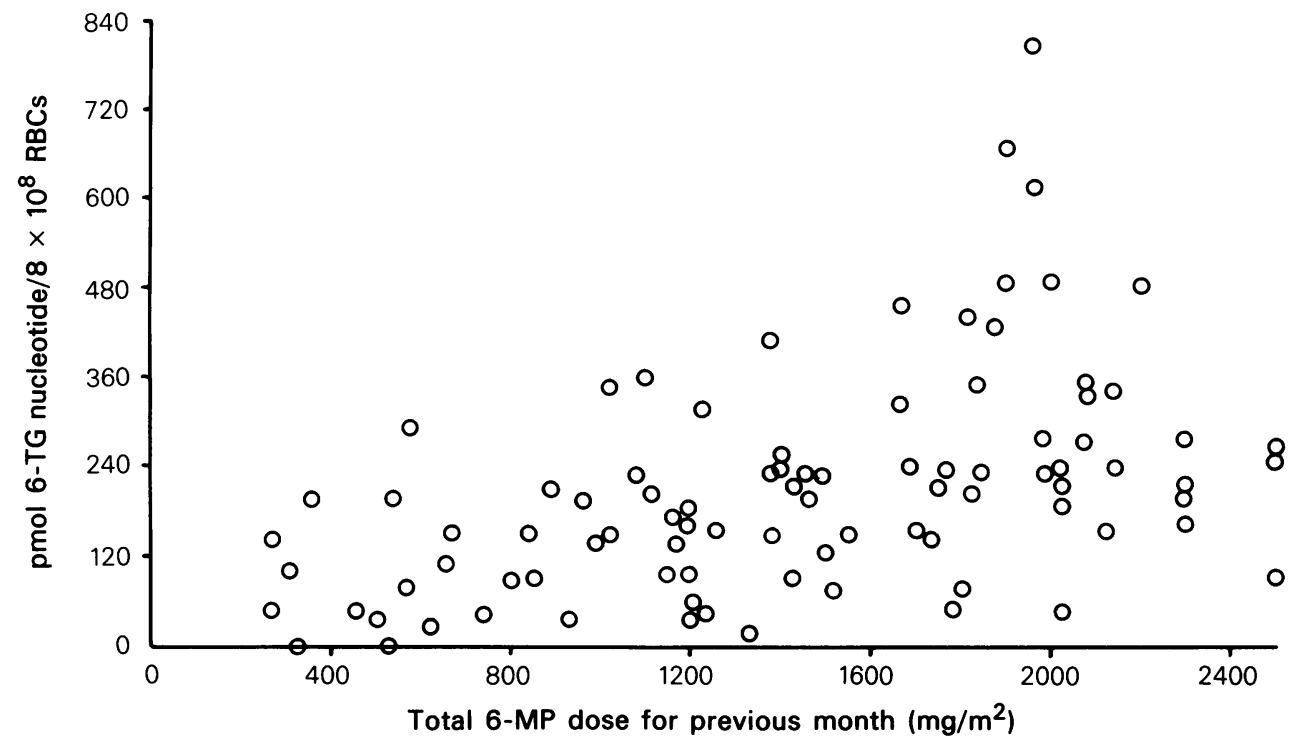

Figure 2 The relationship between total monthly 6-mercaptopurine (6-MP) dose and 6-thioguanine (6-TG) nucleotide concentration $(r=0.48 ; P<0.001 ; n=90 ; y=6.49( \pm 5.98)+0.0198( \pm 0.0039) x)$. The $95 \%$ confidence limits for the regression coefficient $b$ are 0.0122 to 0.0274 .

limits for the regression coefficient $b$ are -0.0054 to -0.0266 . The RBC 6-TG nucleotide concentration (day 0) also correlated with the ANC at day $14(r=$ $-0.3 ; P<0.01 ; n=90)$. The $95 \%$ confidence limits for $r=-0.3$ are -0.1 to -0.48 .

If the data obtained on occasions when other, uncontrolled, factors may have been influencing haematopoiesis, i.e. co-trimoxazole therapy (Asmar et al., 1981), folate deficiency or proven infections, ' were excluded from the analysis the correlation between RBC 6-TG nucleotide concentration and neutropenic effect at day 14 increased $(r=-0.6 ; P<0.001 ; n=$ 37) (Figure 3). The correlation between 6-MP dose (day 0$)$ and ANC at day 14 also improved $(r=-0.5 ; P$ $<0.01 ; n=37 ; \mathrm{y}=3.89( \pm 0.52)-0.034( \pm 0.0089) \mathrm{x})$. The $95 \%$ confidence limits for the regression coefficient $b$ are -0.017 to -0.051 .

When this group, not influenced by co-trimoxazole, folate deficiency or infections, was divided about the mean 6-TG nucleotide concentration ( $210 \mathrm{pmol})$, an arbitrary value, there was a highly significant difference $(P<0.001)$ between the mean ANC (day 14) of the resulting two groups. The mean ANC (day 14) of the group with $>210$ pmol 6-TG nucleotide per $8 \times$ $10^{8} \mathrm{RBCs}$ was $1.13 \pm 0.618 \times 10^{9} \mathrm{I}^{-1}(n=16)$ indicating a significant degree of neutropenia in this group. The mean ANC (day 14) for the group $<210$ pmol 6-TG nucleotide per $8 \times 10^{8} \mathrm{RBCs}$ was $2.88 \pm 1.73 \times$ $10^{9} 1^{-1}(n=21)$.

If the data obtained on occasions when co-trimoxazole, folate deficiency or infections may have been influencing the ANC were compared to the data not

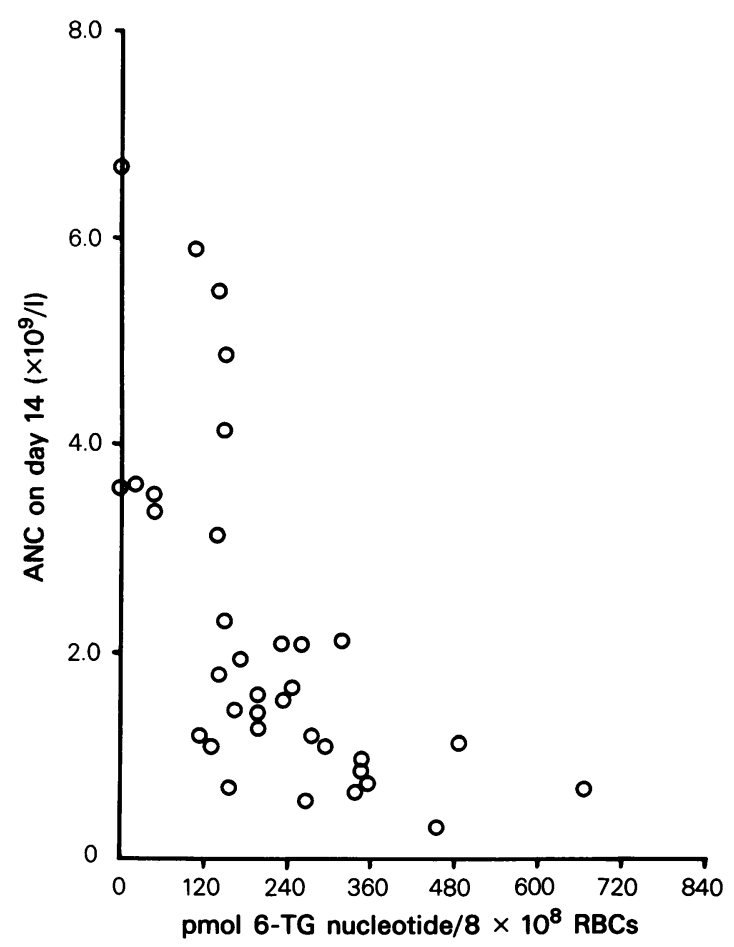

Figure 3 The relationship between red blood cell (RBC) 6-thioguanine (6-TG) nucleotide concentration (day 0) and absolute neutrophil count (ANC) (day 14) in the group not influenced by co-trimoxazole, folate deficiency or proven infections $(r=-0.6 ; P<0.001 ; n=$ 37). The $95 \%$ confidence limits for $r=-0.6$ are -0.35 to -0.77 . 
influenced by these variables there was no statistical difference between the mean 6-MP dose or 6-TG nucleotide concentration for these two groups. There was a statistically significant difference $(P<0.01)$ between the mean 14 day ANC for these two groups $\left(1.24 \pm 1.08 \times 10^{9} \mathrm{l}^{-1}, n=53\right.$ and $2.13 \pm 1.66 \times 10^{9} \mathrm{l}^{-1}$, $n=37$, respectively).

\section{Metabolite accumulation}

At the start of 6-MP therapy 6-TG nucleotide accumulated slowly within the RBC (Figure 4). Four children were studied on similar 6-MP daily doses and both the rate and amount of accumulation varied. One reached and two approached steady state, the fourth child showed linear accumulation. Dose adjustment, due to neutropenia, occurred on days 14, 21 and 28 for those children taking $6-\mathrm{MP}$ at 67,62 and $79 \mathrm{mg} / \mathrm{m}^{2}$, respectively.

In the group of 22 studied two children had low 6-TG nucleotide concentrations $\left(<90 \mathrm{pmol} / 8 \times 10^{8}\right.$ RBC) when taking continuous high dose 6-MP. Neutropenia was not observed.

\section{Metabolite concentration}

The concentration of RBC 6-TG nucleotide was not related to red cell size or haematocrit. The haemoglobin $(\mathrm{Hb})$ concentration at the time of sampling (day 0 ) was taken as a reflection of the size of the RBC compartment. Correcting the RBC 6-TG nucleotide concentration for compartment size (pmol 6-TG nucleotide per $8 \times 10^{8} \mathrm{RBCs} \times \mathrm{Hb} \mathrm{g} 100 \mathrm{ml}^{-1}$ ) did not alter the relationship between metabolite concentra-

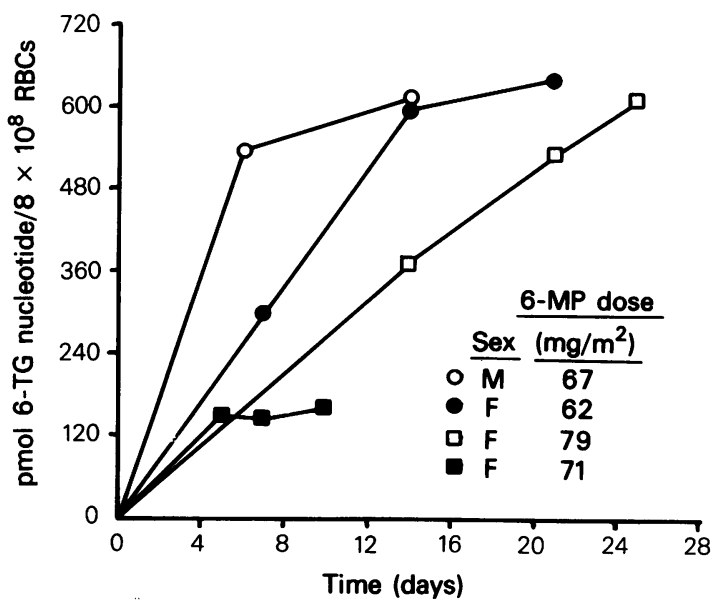

Figure 4 The accumulation of 6-thioguanine (6-TG) nucleotide within the red blood cell (RBC) from the start of 6-mercaptopurine (6-MP) maintenance therapy. Throughout the measurements the patient's daily dose was constant. tion and ANC at day 14. The correlation coefficients for the whole group and for the group not influenced by co-trimoxazole, folate deficiency or infections were $r=-0.31(n=90)$ and $r=-0.6(n=37)$, respectively.

\section{Discussion}

The correlation between RBC 6-TG nucleotide concentration and neutropenia, observed in the pilot study, has been confirmed. The pilot study, consisting of 22 samples taken from 15 children with lymphoblastic leukaemia, showed no correlation between dose and metabolite or dose and neutropenia. These children were on earlier MRC trials (UKALL V, VI or VII) where 6-MP was either taken continuously throughout remission or for 3 weeks out of 4. 6-MP dose was not titrated to neutropenia as aggressively as in UKALL VIII. This, and the small number of samples, could account for the previously observed lack of relation between 6-MP dose and biological effect.

The present study consisted of repeat samples taken over a course of 6-MP treatment, which included periods of dose adjustment, during remission. The continual adjustment of 6-MP dose means that children are not necessarily at steady state.

6-MP dose was significantly correlated to RBC 6TG nucleotide but large individual variations around the regression line are observed. When 6-MP daily dose is plotted against the 6-TG nucleotide concentration (Figure 1) several samples contained nucleotide when no 6-MP had been taken for the previous week. 6-TG nucleotide was not detected in children who had never taken 6-MP. This indicates a long half-life for the nucleotide metabolite, measured in days rather than hours. The improved correlation observed between the total monthly dose and nucleotide metabolite concentration indicates that intracellular 6-TG nucleotide reflects the metabolism of a whole course of treatment rather than a single 6-MP dose.

We have little information on the intermediate metabolism of 6-MP leading to the formation of 6-TG nucleotide in the RBC. The relationships between this metabolite and neutropenia could indicate that red cell metabolites are in some sort of equilibrium with the bone marrow. Alternatively, RBC 6-TG nucleotide could be a reflection of the cytotoxically active nucleotide within the bone marrow stem cells.

There are other variables which may influence the ANC and therefore the relationship with 6-MP and 6-TG nucleotides. The bone marrow will also be affected by the other cytotoxic drugs which, in addition to 6-MP, are used in remission maintenance therapy. Relapse of the disease itself could result in a reduced number of neutrophils in peripheral blood. In some children other uncontrolled potentially myelosup- 
pressive factors (co-trimoxazole therapy, folate deficiency, proven infections), could have been influencing haemotopoiesis. Thus, using the ANC, there may be a tendency to adjust the 6-MP dose in response to neutropenia produced by other factors. However, there is less tendency to increase 6-MP dose above a standard level if the ANC remains normal. In some children haematological relapse may be due to doses that are sub-optimal because of interindividual differences in pharmacokinetics.

In childhood lymphoblastic leukaemia the measure-

\section{References}

ASMAR, B.I., MAQBOOL, S. \& DAJANI, A.S. (1981). Hematologic abnormalities after oral trimethoprimsulfamethoxazole therapy in children. Am. J. Dis. Child., 135, 1100-1103.

HERBER, S., LENNARD, L., LILLEYMAN, J.S. \& MADDOCKS, J. (1982). 6-Mercaptopurine: Apparent lack of relation between prescribed dose and biological effect in children with leukaemia. Br. J. Cancer, 46, 138-141.

LENNARD, L. \& MADDOCKS, J.L. (1983a). Assay of 6-thioguanine nucleotide, a major metabolite of azathioprine, 6-mercaptopurine and 6-thioguanine in human red cells. J. Pharm. Pharmac., 35, 15-18. ment of RBC 6-TG nucleotide can be used as an index of cytotoxic activity of 6-MP. In the group of children unaffected by co-trimoxazole, folate deficiency or infections neutropenia can be predicted by day 14 if the RBC 6-TG nucleotide at day 0 is $>210$ pmol per $8 \times$ $10^{8} \mathrm{RBCs}$. In those children who fail to respond to 6-MP the assay of 6-TG nucleotide may enable drug metabolism to be identified as a causative factor.

We wish to thank the MRC for financial support to L.L.

LENNARD, L., REES, C., LILLEYMAN, J.S. \& MADDOCKS, J.L. (1983b). 6-Mercaptopurine nucleotide metabolites concentration-response relationship in childhood leukaemia. Br. J. clin. Pharmac., 16, 592P-593P.

TIDD, D.M. \& PATERSON, A.R.P. (1974). A biochemical mechanism for the delayed cytotoxic reaction of 6-mercaptopurine. Cancer Res., 34, 738-746.

(Received March 16, 1983, accepted May 24, 1983) 